\title{
Susceptibility of Anopheles campestris-like and Anopheles barbirostris species complexes to Plasmodium falciparum and Plasmodium vivax in Thailand
}

\author{
Sorawat Thongsahuan', Visut Baimai², Anuluck Junkum¹, Atiporn Saeung1, Gi-Sik Min³, \\ Deepak Joshi' ${ }^{3}$, Mi-Hyun Park ${ }^{3}$, Pradya Somboon', Wannapa Suwonkerd ${ }^{4}$, \\ Pongsri Tippawangkosol ${ }^{1}$, Narissara Jariyapan ${ }^{1}$, Wej Choochote ${ }^{1 /+}$ \\ 'Department of Parasitology, Faculty of Medicine, Chiang Mai University, Chiang Mai, Thailand \\ ${ }^{2}$ Department of Biology and Centre for Vectors and Vector-Borne Diseases, Faculty of Science, Mahidol University, Bangkok, Thailand \\ ${ }^{3}$ Department of Biological Sciences, Inha University, Incheon, South Korea ${ }^{4}$ Office of Vector Borne Diseases Control, \\ Department of Communicable Disease Control, Ministry of Public Health, Chiang Mai, Thailand
}

Nine colonies of five sibling species members of Anopheles barbirostris complexes were experimentally infected with Plasmodium falciparum and Plasmodium vivax. They were then dissected eight and 14 days after feeding for oocyst and sporozoite rates, respectively, and compared with Anopheles cracens. The results revealed that Anopheles campestris-like Forms E (Chiang Mai) and F (Udon Thani) as well as An. barbirostris species A3 and A4 were non-potential vectors for $\mathrm{P}$. falciparum because $0 \%$ oocyst rates were obtained, in comparison to the 86.67-100\% oocyst rates recovered from An. cracens. Likewise, An. campestris-like Forms E (Sa Kaeo) and F (Ayuttaya), as well as An. barbirostris species A4, were non-potential vectors for $\mathrm{P}$. vivax because $0 \%$ sporozoite rates were obtained, in comparison to the 85.71-92.31\% sporozoite rates recovered from An. cracens. An. barbirostris species A1, A2 and $A 3$ were low potential vectors for $\mathrm{P}$. vivax because $9.09 \%, 6.67 \%$ and $11.76 \%$ sporozoite rates were obtained, respectively, in comparison to the 85.71-92.31\% sporozoite rates recovered from An. cracens. An. campestris-like Forms $B$ and E (Chiang Mai) were high-potential vectors for $\mathrm{P}$. vivax because $66.67 \%$ and $64.29 \%$ sporozoite rates were obtained, respectively, in comparison to 90\% sporozoite rates recovered from An. cracens.

Key words: Anopheles barbirostris complexes - malaria susceptibility - Plasmodium falciparum - Plasmodium vivax

Malaria is a major concern in international public health. It is endemic in more than 109 countries and threatens the health of about $50 \%$ of the world's population (350-500 million people/year), particularly in tropical and subtropical regions (e.g., parts of Africa, Asia, the Middle East, Eastern Europe, Central and South America, Hispaniola and Oceania). The disease is caused by six species of Plasmodium and anopheline mosquitoes are important vectors (WHO 1997, Singh et al. 2004, Vythilingam et al. 2006, Sutherland et al. 2010). Five species of malaria parasites are found in Thailand. The most common are Plasmodium vivax Grassi and Feletti (52.70\%) and Plasmodium falciparum Welch (46.74\%) and $0.51 \%$ are mixed infections. Plasmodium malariae Grassi and Feletti $(0.04 \%)$ and Plasmodium ovale Stephens $(0.01 \%)$ are rare and only four cases of Plasmodium knowlesi Sinton and Mulligan have been reported (Jongwutiwes et al. 2004, MPH 2009). Regarding $P$. ovale, based on DNA samples from Ghana, Myanmar, Nigeria, Sao Tome, Sierra Leone and Uganda, at least

Financial support: TRF/BIOTEC/BRT (R252005), TRF/RGJ-Ph.D. (PHD/0082/2549)

+ Corresponding author:wchoocho@mail.med.cmu.ac.th

Received 30 June 2010

Accepted 8 December 2010 two distinct new species, i.e., P. ovale curtisi (classic type) and $P$. ovale wallikeri (variant type), have been described recently by Sutherland et al. (2010). However, the identity of these two new species in Thailand is still ambiguous and needs further detailed investigation. The disease in Thailand is generally limited to rural communities living in or near forested regions, mountains and foothills, particularly in areas near and along its borders with neighbouring countries, i.e., Cambodia, Laos, Myanmar and Malaysia.

There are at least 20 anopheline species playing an important role as primary, secondary and suspected vectors of malaria in Thailand. The primary vectors are Anopheles dirus Peyton and Harrison, Anopheles baimaii Sallum and Peyton, Anopheles minimus s.l. and Anopheles maculatus Theobald, while Anopheles aconitus Doenitz, Anopheles pseudowillmori Theobald and Anopheles epiroticus Linton and Harbach are considered secondary vectors (Gould et al. 1967, Scanlon et al. 1968, Harrison 1980, Rosenberg et al. 1990, Green et al. 1991, Rattanarithikul et al. 1996, Subbarao 1998, Linton et al. 2005, Sallum et al. 2005a, b). The remaining 13 species, Anopheles annularis Van der Wulp, Anopheles barbirostris Van der Wulp, Anopheles campestris Reid, Anopheles karwari James, Anopheles kochi Doenitz, Anopheles nigerrimus Giles, Anopheles nivipes Theobald, Anopheles peditaeniatus Leicester, Anopheles philippinensis Ludlow, Anopheles sawadwongporni Rattanarithikul and Green, Anopheles sinensis Wiedemann, Anopheles tessellatus Theobald and 
Anopheles vagus Doenitz, are suspected vectors based on positive enzyme linked immunosorbent assay results for oocysts in the midgut and/or circumsporozoite antigens (Baker et al. 1987, Harbach et al. 1987, Gingrich et al. 1990, Frances et al. 1996, Rattanarithikul et al. 1996).

As early as 1953, the An. barbirostris/campestris group was considered a suspected vector of malaria and/ or filariasis in Thailand (Iyengar 1953, Griffith 1955, Scanlon et al. 1968). The group was proven to be a natural vector of malaria because of the $P$. vivax and filariasis caused by Brugia malayi Brug and Brugia timori Partono in Malaysia and Indonesia (Reid 1968, Atomosoedjono et al. 1976, Kirnowardoyo 1985). Recently, members of the An. barbirostris/campestris group were incriminated as potential natural vectors of $P$. vivax in the Aranyaprathet district, Sa Kaeo province, eastern Thailand (Limrat et al. 2001, Apiwathnasor et al. 2002). In addition, they were also considered vectors that played an important role in the increase in cases of $P$. vivax infection in Thailand (Sattabongkot et al. 2004).

Differences in malarial vector-competence among sibling species members of some Anopheles species complexes have been noticed, particularly in determining their potentiality as vectors, by comparative susceptibility tests to malaria parasites under laboratory conditions. As determined by sporozoite rates in the salivary glands of Anopheles culicifacies Giles complexes, species A and C were susceptible to $P$. vivax, while species B was refractory (Subbarao 1998, Adak et al. 1999). Species A was the most susceptible, whereas species B was the least susceptible species to both Plasmodium yoelii yoelii Landau and Killick-Kendrick and Plasmodium vinckei petteri Carter and Walliker (Kaur et al. 2000). In the Anopheles oswaldoi complexes, An. oswaldoi Peryassú was susceptible to P. vivax, while Anopheles konderi Galvão and Damasceno was refractory (Marrelli et al. 1999). Recently, at least five sibling species have been discovered within the $A n$. barbirostris/campestris group, i.e., An. campestris-like and An. barbirostris species A1, A2, A3 and A4 (Saeung et al. 2007, 2008, Suwannamit et al. 2009). Little is known about the potential vector for $P$. falciparum and/or $P$. vivax in these five sibling species members; thus, intensive comparison of their vector-competence is needed to elucidate their potential vector status. This study reports the susceptibility in the laboratory of An. campestris-like forms and An. barbirostris species A1, A2, A3 and A4 to P. falciparum and P. vivax.

\section{SUBJECTS, MATERIALS AND METHODS}

Laboratory-raised isoline colonies - Nine isoline colonies, An. campestris-like Forms B (Chiang Mai), E (Chiang Mai \& Sa Kaeo) and F (Ayuttaya \& Udon Thani) and An. barbirostris species A1 (Chiang Mai), A2 (Phetchaburi), A3 (Kanchanaburi) and A4 (Chiang Mai) were established based on morphological, cytogenetic and molecular investigations and crossing experiments, as previously reported by Saeung et al. $(2007,2008)$, Suwannamit et al. (2009) and Thongsahuan et al. (2009). These colonies were successfully reared using the methods of Choochote et al. (1983) and Kim et al. (2003) in an insectary room at $27 \pm 2^{\circ} \mathrm{C}$ and $70-80 \%$ relative humid- ity $(\mathrm{RH})$. The room was illuminated with a combination of natural daylight from glass windows and fluorescent lighting (approximately $12 \mathrm{~h}$ per day) and the malarial susceptibility test was used throughout the experiments. Anopheles cracens Sallum and Peyton, formerly known as Anopheles dirus B, has been proven to be an efficient laboratory vector for both $P$. falciparum and $P$. vivax (Junkum et al. 2005, Sallum et al. 2005a, b) and was used as the control vector in the malarial susceptibility experiments. The colony of An. cracens was originally obtained from the Armed Forces Research Institute of Medical Sciences, Bangkok, Thailand, and the freemating colony (Sucharit \& Choochote 1983) had been established for more than two decades in the insectary of the Department of Parasitology, Faculty of Medicine, Chiang Mai University, Chiang Mai, Thailand.

P. falciparum and P. vivax gametocytes - The gametocytes of $P$. falciparum and $P$. vivax were obtained from malaria patients who were infected in Maetang and/or other districts in Chiang Mai province, northern Thailand. Ten millilitres of blood containing gametocytes from the two malaria species was collected by venipuncture in a heparinized syringe, kept at ambient temperature and used for infecting mosquitoes within $12 \mathrm{~h}$ of collection. Informed consent was obtained from the patients before blood collection and the study protocols were approved by the Internal Review Board of Chiang Mai Office for Vector Borne Diseases Control, Department of Communicable Disease Control, Ministry of Public Health, Thailand.

Infection of mosquitoes with $P$. falciparum and $P$. vivax gametocytes - After emergence, all adult female mosquitoes were provided with a $10 \%$ sucrose solution until they were 4-6 days old. They were subsequently fasted for $12 \mathrm{~h}$ prior to being infected. The 12-h fasted females of An. campestris-like forms, An. barbirostris species A1, A2, A3 and A4 and a control mosquito-vector (An. cracens) were put in paper cups that were $8.5 \mathrm{~cm}$ in diameter and $11 \mathrm{~cm}$ deep (50 fasted females per cup for each species) and allowed to feed on heparinised blood containing gametocytes using the artificial membrane feeding techniques described by Chomcharn et al. (1980). The different groups of fasted females belonging to distinct species and/or forms were not always fed simultaneously with the same infective blood. After feeding, the fully engorged females were separated into smaller paper cups (diameter $6.5 \mathrm{~cm}$, depth $8 \mathrm{~cm}$ ) containing 10 mosquitoes per cup. The cups were maintained in an insectary at $27 \pm 2^{\circ} \mathrm{C}$ and $70-80 \%$ RH. A cotton wool pad soaked with $10 \%$ sucrose solution was provided regularly and changed every other day until the time of dissection. Eight and 14 days after feeding, the infected mosquitoes were dissected and examined for oocysts in the midgut and sporozoites in the salivary glands.

\section{RESULTS}

Infection of mosquitoes with P. falciparum gametocytes - Details of the oocyst and sporozoite rates of An. cracens, An. campestris-like Forms $\mathrm{E}$ and $\mathrm{F}$ and An. barbirostris species A3 and A4 eight and 14 days post-infection with $P$. falciparum gametocytes are shown in Table I. 
The $100 \%$ and $86.67 \%$ oocyst rates corresponded to an average of 114.80 and 86.38 oocysts per infected midgut eight and 14 days after feeding, respectively. The 93.33\% sporozoite rate obtained from An. cracens, an efficient control vector, 14 days after feeding, showed that the feedings were conditional on the proper density and maturity of the infective gametocytes in infected blood. The oocyst and sporozoite rates recovered from An. campestris-like Forms E (Chiang Mai) and F (Udon Thani) and An. barbirostris species A3 and A4 were $0 \%$ at both eight and 14 days after feeding.

Infection of mosquitoes with $P$. vivax gametocytes - Details of the oocyst and sporozoite rates of $A n$. cracens, An. campestris-like Forms B, E and F and An. barbirostris species A1, A2, A3 and A4 eight and 14 days post-infection with $P$. vivax gametocytes are shown in Table II. Pictures of oocyst-infected midguts and sporozoite-infected salivary glands of An. cracens and An. campestris-like Form E are illustrated in Figs 1 and 2 . The $100 \%, 100 \%$ and $100 \%$ oocyst rates corresponded to an average of $108.60,145.00$ and 10.20 oocysts per infected midgut in experiments I, II and III, respectively, eight days after feeding. The $90 \%, 85.71 \%$ and $76.92 \%$ oocyst rates corresponded to an average of $48.44,40.17$ and 4.50 oocysts per infected midgut in experiments I, II and III, respectively, 14 days after feeding. The $90 \%, 85.71 \%$ and $92.31 \%$ sporozoite rates in experiments I, II and III, respectively, were obtained 14 days after feeding from An. cracens, an efficient control vector. These rates exhibited that all feedings were conditional on the proper density and maturity of infective gametocytes in infected blood.

An. campestris-like forms (Chiang Mai) (experiment I) - Comparative statistical analyses of the oocyst rates and average number of oocysts per infected midgut of An. campestris-like forms (Chiang Mai) [oocyst rates: Forms B (100\% and 66.67\%) and E (100\% and $100 \%)$,
8 and 14 days, respectively, after feeding; average number oocyst per infected midgut: Forms B (77.60 and 20.75) and E (131 and 42), 8 and 14 days, respectively, after feeding] and An. cracens, an efficient control vector, exhibited no significant difference in all experimental studies $(\mathrm{p}>0.05)$. Likewise, the sporozoite rates of An. campestris-like forms [Forms B (66.67\%) and E (64.29\%), 14 days after feeding] did not differ significantly from An. cracens ( $\mathrm{p}>0.05)$.

An. campestris-like Forms E (Sa Kaeo) and F (Ayuttaya) - The oocyst rates of An. campestris-like Forms E [experiment II (60\%) and III (40\%), 8 days after feeding; experiment II (33.33\%) and III (0\%), 14 days after feeding] and $\mathrm{F}$ [experiment II (40\%) and III (20\%), 8 days after feeding; experiment II (12.50\%) and III (0\%), 14 days after feeding] and the average number of oocysts per infected midgut of An. campestris-like Forms E [experiment II (20.33) and III (2.50), 8 days after feeding; experiment II (12.20) and III (0), 14 days after feeding] and $\mathrm{F}$ [experiment II (8.50) and III (1.50), 8 days after feeding; experiment II (2.50) and III (0), 14 days after feeding] were lower than An. cracens, an efficient control vector, in all experimental studies. Comparative statistical analyses of the oocyst rates and average number of oocysts per infected midgut between An. cracens and An. campestris-like Forms $\mathrm{E}$ and $\mathrm{F}$, eight days after feeding, were performed. The results demonstrated that only the oocyst rates between An. cracens and An. campestris-like Form F (experiment III) differed significantly $(\mathrm{p}<0.05)$, whereas only the average number of oocysts per infected midgut between An. cracens and An. campestris-like Forms $\mathrm{E}$ and $\mathrm{F}$ (experiment III) did not differ significantly $(\mathrm{p}>0.05)$. Comparative statistical analyses of the oocyst rates and average number of oocysts per infected midgut between An. cracens and An. campestris-like Forms E and F, 14 days after feeding, were not done because during this period the mature oocysts from the midguts of An. cracens ruptured and

\section{TABLE I}

Oocysts and sporozoites detected from Anopheles cracens, Anopheles campestris-like Forms E and F and Anopheles barbirostris species A3 and A4 eight and 14 days post infection with Plasmodium falciparum ${ }^{a}$

\begin{tabular}{|c|c|c|c|c|c|}
\hline \multirow[b]{2}{*}{ Species } & \multicolumn{2}{|r|}{8 days } & \multicolumn{3}{|c|}{14 days } \\
\hline & $\begin{array}{c}\text { Oocyst rate } \\
\text { (n) }\end{array}$ & $\begin{array}{l}\text { Average number of } \\
\text { oocysts per infected } \\
\text { midgut (range) }\end{array}$ & $\begin{array}{l}\text { Oocyst rate } \\
\text { (n) }\end{array}$ & $\begin{array}{l}\text { Average number of } \\
\text { oocysts per infected } \\
\text { midgut (range) }\end{array}$ & $\begin{array}{l}\text { Sporozoite rate } \\
\text { (n) }\end{array}$ \\
\hline An. cracens & $100(5 / 5)$ & $114.80 \pm 25.26(79-141)$ & $86.67(13 / 15)$ & $86.38 \pm 65.39(4-201)$ & $93.33(14 / 15)$ \\
\hline \multicolumn{6}{|c|}{ An. campestris-like forms } \\
\hline E (Chiang Mai) & $0(0 / 5)$ & 0 & $0(0 / 14)$ & 0 & $0(0 / 14)$ \\
\hline F (Udon Thani) & $0(0 / 5)$ & 0 & $0(0 / 12)$ & 0 & $0(0 / 12)$ \\
\hline \multicolumn{6}{|c|}{ An. barbirostris species } \\
\hline A3 & $0(0 / 5)$ & 0 & $0(0 / 17)$ & 0 & $0(0 / 17)$ \\
\hline A4 & $0(0 / 5)$ & 0 & $0(0 / 11)$ & 0 & $0(0 / 11)$ \\
\hline
\end{tabular}

a: gametocyte density $/ 200 \mathrm{wbc}=40$. 
TABLE II

Oocysts and sporozoites detected from Anopheles cracens, Anopheles campestris-like Forms B, E and F and Anopheles barbirostris species A1, A2, A3 and A4 eight and 14 days post infection with Plasmodium vivax ${ }^{\mathrm{a}}$

\begin{tabular}{|c|c|c|c|c|c|}
\hline \multirow[b]{2}{*}{ Species } & \multicolumn{2}{|r|}{8 days } & \multicolumn{3}{|c|}{14 days } \\
\hline & $\begin{array}{l}\text { Oocyst rate } \\
\text { (n) }\end{array}$ & $\begin{array}{l}\text { Average number of } \\
\text { oocysts per infected } \\
\text { midgut (range) }\end{array}$ & $\begin{array}{l}\text { Oocyst rate } \\
\text { (n) }\end{array}$ & $\begin{array}{l}\text { Average number of } \\
\text { oocysts per infected } \\
\text { midgut (range) }\end{array}$ & $\begin{array}{l}\text { Sporozoite rate } \\
\text { (n) }\end{array}$ \\
\hline \multicolumn{6}{|l|}{ Experiment I } \\
\hline An. cracens & $100(5 / 5)$ & $108.60 \pm 38.22(53-136)$ & $90(18 / 20)$ & $48.44 \pm 17.84(17-78)$ & $90(18 / 20)$ \\
\hline \multicolumn{6}{|c|}{ An. campestris-like Forms } \\
\hline B (Chiang Mai) & $100(5 / 5)$ & $77.60 \pm 37.33(29-130)$ & $66.67(4 / 6)$ & $20.75 \pm 9.91(8-32)$ & $66.67(4 / 6)$ \\
\hline E (Chiang Mai) & $100(5 / 5)$ & $131 \pm 42.95(91-194)$ & $100(14 / 14)$ & $42 \pm 14.90(14-77)$ & $64.29(9 / 14)$ \\
\hline \multicolumn{6}{|c|}{ An.barbirostris species } \\
\hline A2 & $40(2 / 5)$ & $18 \pm 4.24(15-21)^{c}$ & $26.67(4 / 15)$ & $7.75 \pm 2.63(4-10)$ & $6.67(1 / 15)^{d}$ \\
\hline A3 & $40(2 / 5)$ & $13.50 \pm 6.36(9-18)^{c}$ & $29.41(5 / 17)$ & $3.20 \pm 1.92(1-6)$ & $11.76(2 / 17)^{d}$ \\
\hline \multicolumn{6}{|l|}{ Experiment II } \\
\hline An. cracens & $100(5 / 5)$ & $145 \pm 93.37(11-245)$ & $85.71(18 / 21)$ & $40.17 \pm 66.38(1-248)$ & $85.71(18 / 21)$ \\
\hline \multicolumn{6}{|c|}{ An. campestris-like Forms } \\
\hline E (Sa Kaeo) & $60(3 / 5)$ & $20.33 \pm 16.44(8-39)^{c}$ & $33.33(5 / 15)$ & $12.20 \pm 9.73(3-24)$ & $0(0 / 15)$ \\
\hline F (Ayuttaya) & $40(2 / 5)$ & $8.50 \pm 4.95(5-12)^{c}$ & $12.50(2 / 16)$ & $2.50 \pm 2.12(1-4)$ & $0(0 / 16)$ \\
\hline \multicolumn{6}{|c|}{ An. barbirostris species } \\
\hline A1 & $60(3 / 5)$ & $20.33 \pm 11.50(9-32)^{c}$ & $27.27(6 / 22)$ & $3.83 \pm 2.48(1-8)$ & $9.09(2 / 22)^{d}$ \\
\hline A4 & $40(2 / 5)$ & $5.50 \pm 2.12(4-7)^{c}$ & $5.56(1 / 18)$ & $2 \pm 0(2)$ & $0(0 / 18)$ \\
\hline \multicolumn{6}{|l|}{ Experiment III } \\
\hline An. cracens & $100(5 / 5)$ & $10.20 \pm 6.46(1-18)$ & $76.92(10 / 13)$ & $4.50 \pm 2.07(1-7)$ & $92.31(12 / 13)$ \\
\hline \multicolumn{6}{|c|}{ An. campestris-like Forms } \\
\hline E (Sa Kaeo) & $40(2 / 5)$ & $2.50 \pm 0.71(2-3)$ & $0(0 / 12)$ & 0 & $0(0 / 12)$ \\
\hline F (Ayuttaya) & $20(1 / 5)^{b}$ & $1.50 \pm 0.71(1-2)$ & $0(0 / 14)$ & 0 & $0(0 / 14)$ \\
\hline
\end{tabular}

a: gametocyte density $/ 200 \mathrm{wbc}=32,15$ and 20 in experiment I, II and III, respectively; $b: \mathrm{p}<0.05$ (Fisher exact test); $c$ : $<0.05$ (t-test, two-sides); $d: \mathrm{p}<0.05$ (Fisher exact test).

yielded unreliable results. Interestingly, different stages of oocyst development could be observed in An. campestris-like Forms E and F compared to An. cracens. Most of the oocysts recovered from An. cracens, eight and 14 days after feeding, showed a mature stage of development with a wheel-shaped pattern of sporozoites inside cysts. In An. campestris-like Forms E and F, all of the investigated oocysts had abnormal development, with retaining stages, and some formed melanin inside cysts. The sporozoite rates of An. campestris-like Forms E and F (experiment II and III) were 0\% 14 days after feeding.

An. barbirostris species A1, A2, A3 and A4 - The oocyst rates of $A n$. barbirostris species A1 [experiment II (60\% and $27.27 \%), 8$ and 14 days, respectively, after feeding], A2 [experiment I (40\% and 26.67\%), 8 and 14 days, respectively, after feeding], A3 [experiment I (40\% and $29.41 \%$ ), 8 and 14 days, respectively, after feeding] and A4 [experiment II (40\% and 5.56\%), 8 and 14 days, respectively, after feeding] and the average number of oocysts per infected midgut of An. barbirostris species A1 [experiment II (20.33 and 3.83), 8 and 14 days, respec- tively, after feeding], A2 [experiment I (18 and 7.75), 8 and 14 days, respectively, after feeding], A3 [experiment I (13.50 and 3.20), 8 and 14 days, respectively, after feeding] and A4 [experiment II (5.50 and 2), 8 and 14 days, respectively, after feeding] were lower than An. cracens, an efficient control vector, in all experimental studies. Comparative statistical analyses of the oocyst rates and average number of oocysts per infected midgut between An. cracens and An. barbirostris species A1, A2, A3 and A4, eight days after feeding, were carried out. The results revealed that the oocyst rates between An. cracens and An. barbirostris species A1, A2, A3 and A4 did not differ significantly $(\mathrm{p}>0.05)$, while the average number of oocysts per infected midgut between An. cracens and An. barbirostris species A1, A2, A3 and A4 differed significantly $(\mathrm{p}<0.05)$. Comparative statistical analyses of the oocyst rates and the average number of oocysts per infected midgut between An. cracens and An. barbirostris species A1, A2, A3 and A4 14 days after feeding were not done because during this period the mature oocysts from the midguts of An. cracens ruptured and yielded unreliable results. A few normally developed 

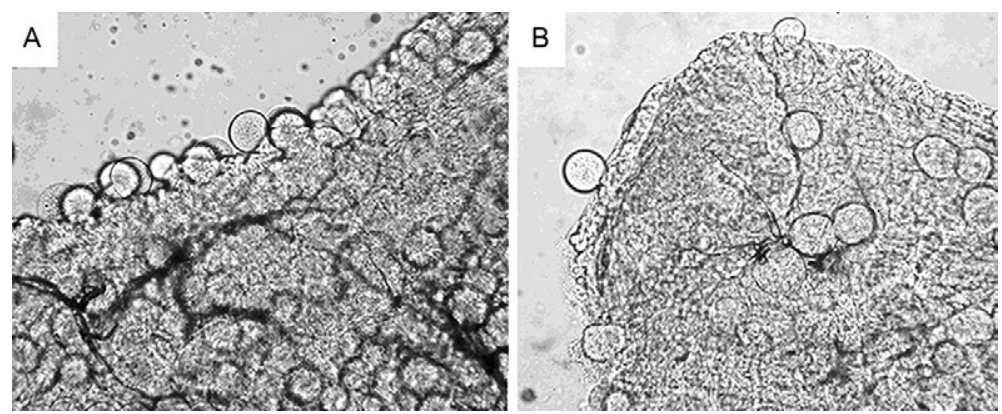

Fig. 1: oocysts of Plasmodium vivax recovering from the midgut of (A) Anopheles cracens and (B) Anopheles campestris-like Form E (Chiang Mai) on day eight after infection.
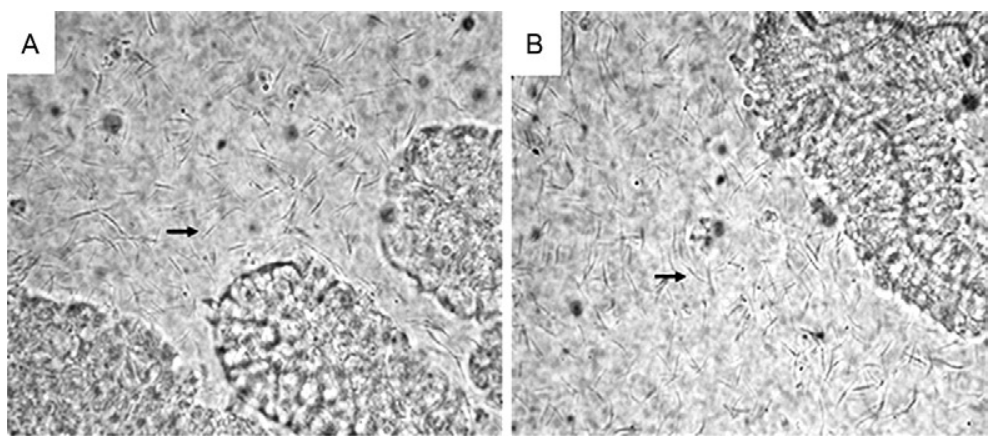

Fig. 2: free flow regular spindle-shaped sporozoites of Plasmodium vivax from the squashed salivary glands (small arrow) of (A) Anopheles cracens and (B) Anopheles campestris-like Form E (Chiang Mai) on day 14 after infection.

oocysts were obtained from An. barbirostris species A1, A2, A3 and A4, particularly eight days after feeding, whereas most of them were abnormally developed and/ or in retaining stages. The sporozoite rates of An. barbirostris species A1 (experiment II), A2 and A3 (experiment I) and A4 (experiment II), 14 days after feedings, were $9.09 \%, 6.67 \%, 11.76 \%$ and $0 \%$, respectively. Comparative statistical analyses of sporozoite rates between An. cracens and An. barbirostris species A1, A2 and A3 differed significantly $(\mathrm{p}<0.05)$.

\section{DISCUSSION}

Before identifying certain mosquitoes as malaria vectors in endemic areas of mosquito-borne human diseases, it is necessary to confirm the susceptibility rate of infection in a laboratory-bred, clean mosquito colony that has been fed on a carrier blood containing pathogens as well as recover the infective stage pathogens in the transmission organ of mosquito vectors. By using this criterion, the susceptibility test in an experimental laboratory is still a useful tool in identifying a certain mosquito species as a potential vector. Nonetheless, susceptibility alone does not imply an important role in the transmission of disease in nature, whereas a refractory result can entirely rule out a vector's significance (Sasa 1976, Rongsriyam et al. 1998, Choochote et al. 2001). In this study, nine isoline colonies of five sibling species members of An. barbirostris complexes, i.e., An. campestris-like Forms B (Chiang Mai), E (Chiang Mai and Sa Kaeo) and F (Udon Thani and Ayut- taya) and An. barbirostris species A1 (Chaing Mai), A2 (Phetchaburi), A3 (Kanchanaburi) and A4 (Chiang Mai) were tested for susceptibility to indigenous strains of $P$. falciparum and $P$. vivax.

For P. falciparum malaria, there was no development of oocysts and there were $0 \%$ sporozoite rates from An. campestris-like Forms E (Chiang Mai) and F (Udon Thani) and An. barbirostris species A3 and A4. This indicated that these species were non-potential vectors for P. falciparum. The results were in agreement with the previous report by Somboon et al. (1994), that showed that An. barbirostris (Mae Hong Son, northern Thailand) was not susceptible to a local strain of $P$. falciparum.

For $P$. vivax malaria, there was abnormal development and/or retaining stage of oocysts with $0 \%$ sporozoite rates from An. campestris-like Forms E (Sa Kaeo) and $\mathrm{F}$ (Ayuttaya) and An. barbirostris species A4. These results revealed that they were non-potential vectors for $P$. vivax. The low normal development of oocysts, with $9.09 \%, 6.67 \%$ and $11.76 \%$ sporozoite rates recovered from An. barbirostris species A1, A2 and A3, respectively, demonstrated that these species were low potential vectors for $P$. vivax. The high normal development of oocysts, with $66.67 \%$ and $64.29 \%$ sporozoite rates, obtained from An. campestris-like Forms B and E (Chiang Mai), respectively, indicated that they were high-potential vectors for $P$. vivax. The above results confirmed that only An. campestris-like and/or An. campestris were potentially natural vectors of $P$. vivax and that these vec- 
tors could be playing an important role in the increasing cases of $P$. vivax in Thailand, as suggested by Harrison and Scanlon (1975), Limrat et al. (2001), Apiwathnasor et al. (2002) and Sattabongkot et al. (2004). Nonetheless, additional investigations of the oocyst and sporozoite rates of wild-caught female An. campestris-like Forms $\mathrm{B}$ and $\mathrm{E}$ in an endemic area of malaria in Chiang Mai province and/or northern Thailand should be intensively carried out to determine the role of this species as a natural vector. Additionally, this study found that there were differences in malarial vector competence among the five sibling species members of An. barbirostris complexes, which confirmed previous reports of different sibling species members. For example, in An. culicifacies complexes, species $\mathrm{A}$ and $\mathrm{C}$ were susceptible to $P$. vivax, while species B was refractory (Subbarao 1998, Adak et al. 1999). Species A was the most susceptible, whereas species B was the least susceptible to both $P$. yoelii yoelii and $P$. vinckei petteri (Kaur et al. 2000). In An. oswaldoi complexes, An. oswaldoi was susceptible to P. vivax, while An. konderi was refractory (Marrelli et al. 1999). Interestingly, the high-potential vectors for $P$. vivax, An. campestris-like Forms B and E (Chiang Mai), were genetically compatible and/or nearly identical to the nonpotential vectors An. campestris-like Forms E (Sa Kaeo) and F (Ayuttaya) (Thongsahuan et al. 2009). Different strains of the same species exhibiting different malarial vector competence has been previously reported, e.g., Anopheles stephensi Liston to Plasmodium gallinaceum Brumpt (Frizzi et al. 1975), Anopheles gambiae Giles to P. gallinaceum (Vernick et al. 1995), Anopheles atroparvus Van Thiel to Plasmodium berghei Vincke and Lips (Sluiters et al. 1986) and An. gambiae to Plasmodium spp (Collins et al. 1986). Competence in these strains appears to be controlled by malaria susceptibility (Pif-B $\left.B^{s} / P i f-B^{r}\right)$ and refractoriness (Pif- $C^{s} /$ Pif- $C^{r}$ ) genes (Vernick et al. 1989). Refractoriness is manifested by malaria mosquito recognition during midgut invasion and the mechanism by which the mosquito defends itself against malaria is induced (Abraham \& Jacobs-Lorena 2004).

The $0 \%$ sporozoite rates recovered from An. campestris-like Form E (Sa Kaeo) in this study were contrary to the $23.80 \%$ sporozoite rate obtained from $A n$. campestris (Sa Kaeo) reported by Apiwathnasor et al. (2002). The morphological criterion of branch summation of seta 2-VI pupal skins used in the identification and/or differentiation of An. campestris-like Form E [Sa Kaeo: 24 (18-31) branches] (Thongsahuan et al. 2009) and An. campestris [Sa Kaeo: 22 (17-58) branches] (Apiwathnasor et al. 2002) from topotypic An. barbirostris (6-18 branches) (Harrison \& Scanlon 1975) was very similar. However, different strains of $P$. vivax gametocyte were used in the susceptibility tests in the different studies; a northern strain (Chiang Mai) was used in this study and an eastern strain (Sa Kaeo) was used by Apiwathnasor et al. (2002). Even though the immunophenotypic strains of P. vivax (VK210 and VK247 variants) (Rosenberg et al. 1989) were not determined in this study, two reports from western and eastern Thailand demonstrated that VK210 was detected in 2/478 An. campestris strains from a western region (Tak) (Coleman et al. 2002) and
VK247 in 2/42 An. barbirostris strains from an eastern area (Chanthaburi) (Frances et al. 1996). The species identification of An. campestris and An. barbirostris in the above studies was based on the morphological criterion of wild-caught adult females that usually exhibit morphological characteristics that are similar across species. These characteristics can cause errors in species identification (Harrison \& Scanlon 1975). Thus, a crucial question is whether the immunophenotypic strains of malaria gametocytes are specifically susceptible to different strains of the An. barbirostris/campestris group. Further detailed investigations of malaria susceptibility and refractoriness genes among allopatric populations of An. campestris-like forms in relation to immunophenotypic strains of $P$. vivax in Thailand are needed to fully understand their malarial vector competence.

\section{ACKNOWLEDGEMENTS}

To Dr Niwes Nantachit, Dean of the Faculty of Medicine, Chiang Mai University, for his interest in this research project.

\section{REFERENCES}

Abraham EG, Jacobs-Lorena M 2004. Mosquito midgut barriers to malaria parasite development. Insect Biochem Mol Biol 34: 667-671.

Adak T, Kaur S, Singh OP 1999. Comparative susceptibility of different members of the Anopheles culicifacies complex to Plasmodium vivax. Trans $R$ Soc Trop Med Hyg 93: 573-577.

Apiwathnasor C, Prommongkol S, Samung Y, Limrat D, Rojruthai B 2002. Potential for Anopheles campestris (Diptera: Culicidae) to transmit malaria parasites in Pa Rai subdistrict (Aranyaprathet, Sa Kaeo province), Thailand. J Med Entomol 39: 583-586.

Atomosoedjono S, Van Peenen PF, Putrali J 1976. Anopheles barbirostris (Van der Wulp) still an efficient vector of Brugia malayi in Central Sulawesi (Celebes), Indonesia. Trans $R$ Soc Trop Med Hyg 70: 259.

Baker EZ, Beier JC, Meek SR, Wirtz RA 1987. Detection and quantification of Plasmodium falciparum and $P$. vivax infections in Thai-Kampuchean Anopheles (Diptera: Culicidae) by enzymelinked immunosorbent assay. J Med Entomol 24: 536-541.

Chomcharn Y, Surathin K, Bunnag D, Sucharit S, Harinasuta T 1980. Effect of a single dose of primaquine on a Thai strain of Plasmodium falciparum. Southeast Asian J Trop Med Public Health 11: 408-412.

Choochote W, Leemingsawat S, Sucharit S, Kunnaphuti J, Wongkamchai S, Sukontason KL, Jitpakdi A 2001. Aedes albolateralis, a potential vector of nocturnally subperiodic Wuchereria bancrofti and dengue type 2 virus. Southeast Asian J Trop Med Public Health 32: 621-624.

Choochote W, Sucharit S, Abeyewickreme W 1983. Experiments in crossing two strains of Anopheles barbirostris van der Wulp 1884 (Diptera: Culicidae) in Thailand. Southeast Asian J Trop Med Public Health 14: 204-209.

Coleman RE, Sithiprasasna R, Kankaew P, Kiaattiut C, Ratanawong S, Khuntirat B, Sattabongkot J 2002. Naturally occurring mixed infection of Plasmodium vivax VK210 and P. vivax VK247 in Anopheles mosquitoes (Diptera: Culicidae) in western Thailand. J Med Entomol 39: 556-559.

Collins FH, Sakai RK, Vernick KD, Paskewitz S, Seeley DC, Miller LH, Collins WE, Campbell CC, Gwadz RW 1986. Genetic selection of a Plasmodium-refractory strain of the malaria vector Anopheles gambiae. Science 234: 607-610. 
Frances SP, Klein TA, Wirtz RA, Eamsila C, Pilakasiri C, Linthicum KJ 1996. Plasmodium falciparum and $P$. vivax circumsporozoite proteins in anophelines (Diptera: Culicidae) collected in eastern Thailand. J Med Entomol 33: 990-991.

Frizzi G, Rinaldi A, Bianchi L 1975. Genetic studies on mechanisms influencing the susceptibility of anopheline mosquitoes to plasmodial infection. Mosquito News 35: 505-508.

Gingrich JB, Weatherhead A, Sattabongkot J, Pilakasiri C, Wirtz RA 1990. Hyperendemic malaria in a Thai village: dependence of yearround transmission on focal and seasonally circumscribed mosquito (Diptera: Culicidae) habitats. J Med Entomol 27: 1016-1026.

Gould DJ, Esah S, Pranith U 1967. Relation of Anopheles aconitus to malaria transmission in the central plain of Thailand. Trans $R S O c$ Trop Med Hyg 61: 441-442.

Green CA, Rattanarithikul R, Pongparit S, Sawadwongporn P, Baimai V 1991. A newly-recognized vector of human malarial parasites in the Oriental region, Anopheles (Cellia) pseudowillmori (Theobald, 1910). Trans R Soc Trop Med Hyg 85: 35-36.

Griffith ME 1955. A note on Anopheles minimus Theobald as a malaria vector in Thailand. VI Intern Congr Microbiol 5: 565-567.

Harbach RE, Gingrich JB, Pang LW 1987. Some entomological observations and malaria transmission in a remote village in northwestern Thailand. J Am Mosq Control Assoc 3: 296-301.

Harrison BA 1980. Medical entomology studies - XIII. The Myzomyia series of Anopheles (Cellia) in Thailand, with emphasis of intra-interspecific variations (Diptera: Culicidae). Contrib $\mathrm{Am}$ Entomol Inst 17: 1-195.

Harrison BA, Scanlon JE 1975. Medical entomology studies - II. The subgenus Anopheles in Thailand (Diptera: Culicidae). Contrib Am Entomol Inst 12: 1-307.

Iyengar MO 1953. Filariasis in Thailand. Bull World Health Organ 9: 731-766.

Jongwutiwes S, Putaporntip C, Iwasaki T, Sata T, Kanbara H 2004. Naturally acquired Plasmodium knowlesi malaria in human, Thailand. Emerg Infect Dis 10: 2211-2213.

Junkum A, Jitpakdi A, Jariyapan N, Komalamisra N, Somboon P, Suwonkerd W, Saejeng A, Bates PA, Choochote W 2005. Susceptibility of two karyotypic forms of Anopheles aconitus (Diptera: Culicidae) to Plasmodium falciparum and P. vivax. Rev Inst Med Trop Sao Paulo 47: 333-338.

Kaur S, Singh OP, Adak T 2000. Susceptibility of species A, B, and C of Anopheles culicifacies complex to Plasmodium yoelii yoelii and Plasmodium vinckei petteri infections. J Parasitol 86: 1345-1348.

Kim SJ, Choochote W, Jitpakdi A, Junkum A, Park SJ, Min GS 2003. Establishment of a self-mating mosquito colony of Anopheles sinensis from Korea. Korean J Entomol 33: 267-271.

Kirnowardoyo S 1985. Status of Anopheles malaria vectors in Indonesia. Southeast Asian J Trop Med Public Health 16: 129-132.

Limrat D, Rojruthai B, Apiwathnasorn C, Samung Y, Prommongkol S 2001. Anopheles barbirostris/campestris as a probable vector of malaria in Aranyaprathet, Sa Kaeo province. Southeast Asian J Trop Med Public Health 32: 739-744.

Linton YM, Dusfour I, Howard TM, Ruiz LF, Duc Manh N, Ho Dinh T, Sochanta T, Coosemans M, Harbach RE 2005. Anopheles (Cellia) epiroticus (Diptera: Culicidae), a new malaria vector species in the southeast Asian sundaicus Complex. Bull Entomol Res 95: 329-339.

Marrelli MT, Honório NA, Flores-Mendoza C, Lourenço-de-Oliveira R, Marinotti O, Kloetzel JK 1999. Comparative susceptibility of two members of the Anopheles oswaldoi complex, An. oswaldoi and An. konderi, to infection by Plasmodium vivax. Trans $R$ Soc Trop Med Hyg 93: 381-384.

MPH - Ministry of Public Health 2009. Division of Malaria 2009. Annual Report, Department of Communicable Disease Control, Ministry of Public Health, Thailand, 78 pp.

Rattanarithikul R, Konishi E, Linthicum KJ 1996. Detection of Plasmodium vivax and Plasmodium falciparum circumsporozoite antigen in anopheline mosquitoes collected in southern Thailand. Am J Trop Med Hyg 54: 114-121.

Reid JA 1968. Anopheline mosquitoes of Malaya and Borneo. Stud Inst Med Res Malaya 31: 1-520.

Rongsriyam Y, Jitpakdi A, Choochote W, Somboon P, Tookyang B, Suwonkerd W 1998. Comparative susceptibility of two forms of Anopheles sinensis Wiedemann 1828 (Diptera: Culicidae) to infection with Plasmodium falciparum, $P$. vivax, $P$. yoelii and the determination of misleading factor for sporozoite identification. Southeast Asian J Trop Med Public Health 29: 159-167.

Rosenberg R, Andre RG, Somchit L 1990. Highly efficient dry season transmission of malaria in Thailand. Trans $R$ Soc Trop Med Hyg 84: 22-28.

Rosenberg R, Wirtz RA, Lanar DE, Sattabongkot J, Hall T, Waters AP, Prasittisuk C 1989. Circumsporozoite protein heterogeneity in the human malaria parasite Plasmodium vivax. Science 245: 973-976.

Saeung A, Baimai V, Otsuka Y, Rattanarithikul R, Somboon P, Junkum A, Tuetun B, Takaoka H, Choochote W 2008. Molecular and cytogenetic evidence of three sibling species of the Anopheles barbirostris Form A (Diptera: Culicidae) in Thailand. Parasitol Res 102: 499-507.

Saeung A, Otsuka Y, Baimai V, Somboon P, Pitasawat B, Tuetun B, Junkum A, Takaoka H, Choochote W 2007. Cytogenetic and molecular evidence for two species in the Anopheles barbirostris complex (Diptera: Culicidae) in Thailand. Parasitol Res 101: 1337-1344.

Sallum MAM, Peyton EL, Harrison BA, Wilkerson RC 2005a. Revision of the Leucosphyrus group of Anopheles (Cellia) (Diptera, Culicidae). Rev Bras Entomol 49 (Suppl. 1): 1-152.

Sallum MAM, Peyton EL, Wilkerson RC 2005b. Six new species of the Anopheles leucosphyrus group, reinterpretation of An. elegans and vector implications. Med Vet Entomol 19: 158-199.

Sasa M 1976. Human filariasis. A global survey of epidemiology and control, University of Tokyo Press, Tokyo, 819 pp.

Sattabongkot J, Tsuboi T, Zollner GE, Sirichaisinthop J, Cui L 2004. Plasmodium vivax transmission: chances for control? Trends Parasitol 20: 192-198.

Scanlon JE, Peyton EL, Gould DJ 1968. An annotated checklist of the Anopheles of Thailand. Thai Natl Sci Pap Fauna Ser 2: 1-35.

Singh B, Kim Sung L, Matusop A, Radhakrishnan A, Shamsul SS, Cox-Singh J, Thomas A, Conway DJ 2004. A large focus of naturally acquired Plasmodium knowlesi infections in human beings. Lancet 363: 1017-1024.

Sluiters JF, Visser PE, van der Kaay HJ 1986. The establishment of Plasmodium berghei in mosquitoes of a refractory and a susceptible line of Anopheles atroparvus. Z Parasitenkd 72: 313-322.

Somboon P, Suwonkerd W, Lines JD 1994. Susceptibility of Thai zoophilic anophelines and suspected malaria vectors to local strains of human malaria parasites. Southeast Asian J Trop Med Public Health 25: 766-770.

Subbarao SK 1998. Anopheline species complexes in South-East Asia. WHO Tech Pub Ser 18: 1-82. 
Sucharit S, Choochote W 1983. Comparative studies on the morphometry of male genitalia and frequency of clasper movements during induced copulation of Anopheles balabacensis (Perlis Form) and Anopheles dirus (Bangkok Colony Strain). Mosq Syst 15: 90-97.

Sutherland CJ, Tanomsing N, Nolder D, Oguike M, Jennison C, Pukrittayakamee S, Dolecek C, Hien TT, do Rosário VE, Arez AP, Pinto J, Michon P, Escalante AA, Nosten F, Burke M, Lee R, Blaze M, Otto TD, Barnwell JW, Pain A, Williams J, White NJ, Day NP, Snounou G, Lockhart PJ, Chiodini PL, Imwong M, Polley SD 2010. Two nonrecombining sympatric forms of the human malaria parasite Plasmodium ovale occur globally. J Infect Dis 201: $1544-1550$.

Suwannamit S, Baimai V, Otsuka Y, Saeung A, Thongsahuan S, Tuetun B, Apiwathnasorn C, Jariyapan N, Somboon P, Takaoka H, Choochote W 2009. Cytogenetic and molecular evidence for an additional new species within the taxon Anopheles barbirostris (Diptera: Culicidae) in Thailand. Parasitol Res 104: 905-918.
Thongsahuan S, Baimai V, Otsuka Y, Saeung A, Tuetun B, Jariyapan N, Suwannamit S, Somboon P, Jitpakdi A, Takaoka H, Choochote W 2009. Karyotypic variation and geographic distribution of Anopheles campestris-like (Diptera: Culicidae) in Thailand. Mem Inst Oswaldo Cruz 104: 558-566.

Vernick KD, Collins FH, Gwadz RW 1989. A general system of resistance to malaria infection in Anopheles gambiae controlled by two main genetic loci. Am J Trop Med Hyg 40: 585-592.

Vernick KD, Fujioka H, Seeley DC, Tandler B, Aikawa M, Miller LH 1995. Plasmodium gallinaceum: a refractory mechanism of ookinete killing in the mosquito, Anopheles gambiae. Exp Parasitol 80: 583-595.

Vythilingam I, Tan CH, Asmad M, Chan ST, Lee KS, Singh B 2006. Natural transmission of Plasmodium knowlesi to humans by Anopheles latens in Sarawak, Malaysia. Trans $R$ Soc Trop Med Hyg 100: 1087-1088.

WHO - World Health Organization 1997. The world health report. Conquering, suffering, enriching humanity, WHO, Geneva, 168 pp. 